\title{
Physicochemical and Microbiological Evaluation of Agbo (herbal decoction) Sold in Some Parts of Warri Metropolis, Delta-State, Nigeria
}

\author{
Ejukonemu E. Francis, Isiosio O. Isaac
}

\begin{abstract}
Agbo which is the Yoruba name for herbal medicine, is an aqueous decoction produced from mixtures of several plant parts such as leaves, stems, roots and barks that is used in treating illnesses and diseases. The study was carried out to ascertain the microbial load, level of heavy metal contamination and antibiotics sensitivity of bacteria isolated from agbo sold in Warri, Delta State. A total of twenty -five (25) agbo samples collected from five (5) locations were analyzed. The $\mathbf{p H}$ values of the various agbo samples ranges from $3.38 \pm$ 1.05 to $7.23 \pm 1.02$. Concentration of Iron ranges from 6.085 \pm $1.00-21.904 \pm 3.04 \mathrm{mg} / \mathrm{l}$, Lead ranges from $0.006 \pm 0.00-0.021 \pm$ $0.08 \mathrm{mg} / \mathrm{l}$, Cadmium ranges from $0.028 \pm 0.10-0.102 \pm 0.11$ $\mathrm{mg} / \mathrm{l}$, Mercury ranges from $0.003 \pm 0.00-0.010 \pm 0.08 \mathrm{mg} / \mathrm{l}$, Arsenic ranges from $0.002 \pm 0.00-0.007 \pm 0.05 \mathrm{mg} / \mathrm{l}$ and Chromium ranges from $0.012 \pm 0.03-0.034 \pm 0.01 \mathrm{mg} / \mathrm{l}$ respectively. The mean total viable count ranges between $25 \pm$ $1.03 \times 104-44 \pm 0.06 \times 104 \mathrm{cfu} / \mathrm{ml}$, total coliforn count ranges between $0.15 \pm 0.51 \times 104-0.30 \pm 0.11 \times 104 \mathrm{cfu} / \mathrm{ml}$ and total fungal count ranges between $25 \pm 1.06-49 \pm 1.05 \times 104 \mathrm{cfu} / \mathrm{ml}$ respectively. S.auerus $(29.4 \%)$ was the predominant bacteria. This was followed by Klebsiella species $(\mathbf{2 3 . 5 \%})$, E.coli $(\mathbf{1 7 . 6 \%})$, Salmonella species $(\mathbf{1 1 . 8 \%})$, Proteus mirabilis (11.8\%) and Enterobacter species (5.9\%). Aspergillus species (23.8) was the predominant fungi. This was followed by Candida species (19.0\%), Penicillium spp (14.3\%), Mucor (14.3\%), Botrytis (9.5\%), Fusarium spp $(9.5 \%)$, Geotrichum spp $(4.8 \%)$ and Phoma spp $(4.8 \%)$. All the isolates were resistant to ampicillin. S.areus was susceptible to augmentin, gentamycin and ceporex, E. coli was susceptible to ciprofloxacin, gentamycin and nalidixic acid. Klebsiella spp was susceptible to ciprofloxacin and getamycin, Salmonella spp was susceptible to ciprofloxacin, P.mirabilis was susceptible to ciprofloxacin and gentamycin, and Enterobacter spp was susceptible to pefloxacine, augmentin and gentamycin.
\end{abstract}

Index Terms - Agbo, Heavy metals, Herbal medicine, Microbial load.

\section{INTRODUCTION}

A herb is a plant or any part of a plant valued for its medicinal, aromatic and savory qualities. Herbal medicine refers to preparations and finished products that contains parts of plant and other plant materials as its active ingredients that is used in treating illness and diseases (Oluyemi et al., 2016). Herbal medicines have been used to treat many conditions such as asthma, eczema, premenstrual syndrome, rheumatoid arthritis, migraine, menopausal

Ejukonemu E. Francis, Department of Science Laboratory Technology, Delta State Polytechnic, Otefe- Oghara

Isiosio O. Isaac, Department of Science Laboratory Technology, Delta State Polytechnic, Otefe- Oghara symptoms, chronic fatigue and irritable bowel syndrome (Falodun et al., 2013)

In Nigeria, the increasing cost of orthodox medication coupled with poverty, has forced a large population to resort to the use of herbal medicines to relief themselves of ailments (Esinome et al., 2001). The use of herbal medicine is prevalent among Nigerians because it is efficient, acts fast, resistant to pathogenic organisms, cheap and readily available (Oluyemi et al., 2016).

Agbo which is the Yoruba name for herbal medicine. It is an aqueous decoction produced from mixture of several plant parts such as leaves, stems, roots and barks. It is prepared by soaking the plants parts in either water or alcohol in bottles and allowed to stand for days or boiling the plant parts after which it is poured into bottles for drinking purpose. The producers and sellers are mostly Yoruba women who hawk the product in Warri and its environs. The product is dispensed in disposable plastic cups to users (Stafford et al., 2008).

Herbal medicines are perceived as being natural and therefore safe; however, they are not free from adverse effects, which may be due to factors such as adulteration, contamination, misidentification, lack of standardization, incorrect preparation and dosage (Oluyege and Oluyege, 2010). By their origin, herbs are subjected to contamination by pathogenic microorganisms from the soil, air and water. In addition, microbial contaminants may also be introduced during harvesting, handling, preparation and storage of herbal medicine. (Ola et al., 2013). Heavy metals such as lead, cadmium, mercury, arsenic, chromium and iron are constituent of the environment like air, water and soil. Furthermore, they are produced by technical and industrial processes, thus medicinal plant growing in nature may bioaccumulate toxic heavy metals to a certain extent depending on their individual properties and concentration of the heavy metals in soil, air and water (Osadolor et al., 2015). In addition, high levels of heavy metals can be found in plants when agricultural expedients such as cadmium fertilizers, organic mercury or lead pesticides are used (Caldas et al., 2004).

Pathogenic microorganisms and heavy metals can occur in herbal medicines when prepared with contaminated herbs. The contamination of these herbal medicines reduces the effectiveness and also poses serious health hazard to the consumers. Heavy metals if consumed can accumulate in different organs of the body leading to unwanted side effects while pathogenic microorganisms cause various diseases. 
(MacDonald, 2015)

The aim of this study was to evaluate the level of heavy metals and microbial contamination of agbo sold in Warri Delta state as well as to test the antibiotics sensitivity of these isolates to commercial antibiotics.

\section{MATERIALS AND METHODS}

\section{A. Collection of samples}

A set of agbo for the treatment of five (5) disease conditions: Typhoid fever, Malaria fever, Erectile dysfunction, general Infections and Rheumatism were randomly purchased at point of sales from sellers at five (5) locations in Warri - Delta State: Okere market, Pessu market, Igbudu market, Effurun market and Main market. $10 \mathrm{ml}$ of each sample was dispensed into sterile containers and taken immediately to the laboratory for analysis.

\section{B. Determination of $\mathbf{p H}$}

The $\mathrm{pH}$ value of each sample was obtained using a $\mathrm{pH}$ meter (search tech PHS - 3C model) at a temperature of $28.5^{\circ} \mathrm{C}$.

\section{Heavy metals analysis}

The amount of heavy metals in each sample was analysed with Electrothermal Atomic Absorption Spectrometer (Perkin Elmer analyst 800, Norwalk, U.S.A) by adopting the methods of Olmedo et al., (2010)

\section{Microbial analysis}

In the laboratory, samples were shaken vigorously to ensure uniform distribution of microorganisms. $5 \mathrm{ml}$ of each sample was pipetted into $95 \mathrm{ml}$ of sterile distilled water for stock sample preparation and was subjected to tenfold serial dilution in sterile test tubes. $1 \mathrm{ml}$ of each stock sample was aseptically transferred and mixed in $9 \mathrm{ml}$ of sterile distilled water $\left(10^{-1}\right)$ up to $10^{-10}$. All media used were prepared according to manufacturer's instructions. For total viable bacterial count and coliform count, $1 \mathrm{ml}$ of dilutions $10^{-2}$ and $10^{-4}$ were transferred into sterile duplicate plates and $15-$ $20 \mathrm{ml}$ of nutrient and MacConkey agar were added and mixed immediately. For fungal count, $1 \mathrm{ml}$ of dilutions $10^{-2}$ and $10^{-4}$ were spread over duplicate plates of pre - prepared dried potato dextrose agar (PDA). Plates were incubated at $32^{\circ} \mathrm{C}$ for 48 hours for total bacterial count, at $37^{\circ} \mathrm{C}$ for 24 hours for coliform count and at $25^{\circ} \mathrm{C}$ for 5 days for fungi count. Plates containing $25-250 \mathrm{CFU} / \mathrm{ml}$ were enumerated for total bacterial count whereas plates containing $15-150 \mathrm{CFU} / \mathrm{ml}$ were enumerated for coliform and fungal count (Christen $e t$ al., 1992). Other media with selective and differential characteristics used were Eosine Methylene Blue (EMB) and Kligler Iron Agar (KIA). Pure cultures bacterial isolates were identified using cultural, morphological and biochemical characterization. Fungal isolates were identified on the basis of macroscopic and microscopic characteristics by slide culture techniques and lactophenol staining. The scheme of Barnet and Hunter (1998) was used for the fungal identification. Bacterial identification to general level was based on Bergey's manual of determinative bacteriology. (Buchanna, and Gibbon, 1974).

\section{E. Antibiotics sensitivity}

Antibiotics sensitivity test was determine by the disc diffusion method as described by Bauer et al. (1966) in accordance with Mcfarland standard. Ten different commercially prepared antibiotics discs containing Ofloxacin $(10 \mu \mathrm{g})$, Pefloxacine $(10 \mu \mathrm{g}), \quad$ Ciprofloxacine $(10 \mu \mathrm{g})$, Augmentin $(30 \mu \mathrm{g})$, gentamcin $(10 \mu \mathrm{g})$, Streptomycin $(30 \mu \mathrm{g})$, Ceporex $(10 \mu \mathrm{g})$, Nalidixic Acid $(30 \mu \mathrm{g})$, Septrin $(30 \mu \mathrm{g})$, and Ampicillin $(30 \mu \mathrm{g})$ were used and after $18 \mathrm{hrs}$ incubation at $37^{\circ} \mathrm{c}$, the size of the zone of inhibition was measured and interpreted by comparing with the standard antibiotic sensitivity chart to determine their resistance patterns.

\section{F. Statistical analysis}

The mean and standard deviation of the results were done using SPSS. The results were expressed as mean \pm S.D. The results were also represented using descriptive statistics such as pie charts.

\section{RESULTS AND DISCUSSIONS}

Table 1: pH values of each samples

\begin{tabular}{lll}
\hline Sample source & Sample type & pH (mean \pm S.D) \\
\hline \multirow{3}{*}{ Main market } & Erectile dysfunction & $7.23 \pm 1.02$ \\
& Rheumatism & $3.79 \pm 0.03$ \\
& Malaria & $4.77 \pm 0.07$ \\
& Typhoid & $4.18 \pm 0.04$ \\
& Infection & $5.83 \pm 1.00$ \\
& & \\
Pessu market & Erectile dysfunction & $4.42 \pm 0.07$ \\
& Rheumatism & $4.58 \pm 0.03$ \\
& Malaria & $5.48 \pm 0.02$ \\
& Typhoid & $4.60 \pm 0.05$ \\
& Infection & $5.53 \pm 1.03$ \\
& & \\
Effurun market & Erectile dysfunction & $5.41 \pm 0.08$ \\
& Rheumatism & $4.68 \pm 0.05$ \\
& Malaria & $4.78 \pm 0.01$ \\
& Typhoid & $4.02 \pm 0.05$ \\
& Infection & $5.88 \pm 1.00$
\end{tabular}




\begin{tabular}{lll} 
& Erectile dysfunction & $3.38 \pm 1.05$ \\
Okere market & Rheumatism & $3.66 \pm 1.03$ \\
& Malaria & $4.87 \pm 0.45$ \\
& Typhoid & $4.26 \pm 0.01$ \\
& Infection & $6.55 \pm 0.08$ \\
Igbudu market & Erectile dysfunction & $3.88 \pm 0.01$ \\
& Rheumatism & $4.65 \pm 0.08$ \\
& Malaria & $5.54 \pm 0.05$ \\
& Typhoid & $4.93 \pm 0.45$ \\
& General Infection & $5.75 \pm 0.07$ \\
\hline
\end{tabular}

Table 2: The Values of heavy metals present in herbal medicine

\begin{tabular}{|c|c|c|c|c|c|c|c|}
\hline \multirow{2}{*}{$\begin{array}{l}\text { Sample } \\
\text { source }\end{array}$} & \multirow[t]{2}{*}{ Sample type } & \multicolumn{6}{|c|}{ Heavy metals (mean \pm S.D Mg/l) } \\
\hline & & Fe & $\mathbf{P b}$ & Cd & $\mathbf{H g}$ & As & $\mathrm{Cr}$ \\
\hline \multirow{11}{*}{$\begin{array}{l}\text { Main } \\
\text { Market }\end{array}$} & \multirow[t]{2}{*}{ Erectile-dysfunction } & $8.452 \pm$ & $0.008 \pm$ & $0.039 \pm$ & $0.004 \pm$ & $0.003 \pm$ & $0.013 \pm$ \\
\hline & & 1.01 & 0.04 & 0.05 & 0.02 & 0.04 & 0.02 \\
\hline & \multirow[t]{2}{*}{ Rheumatism } & $10.333 \pm$ & $0.010 \pm$ & $0.048 \pm$ & $0.005 \pm$ & $0.004 \pm$ & $0.016 \pm$ \\
\hline & & 0.09 & 0.05 & 0.03 & 0.09 & 0.00 & 0.05 \\
\hline & \multirow[t]{2}{*}{ Malaria } & $21.671 \pm$ & $0.021 \pm$ & $0.101 \pm$ & $0.010 \pm$ & $0.007 \pm$ & $0.034 \pm$ \\
\hline & & 2.03 & 0.08 & 0.06 & 0.01 & 0.03 & 0.01 \\
\hline & \multirow[t]{2}{*}{ Typhoid } & $8.397 \pm$ & $0.008 \pm$ & $0.039 \pm$ & $0.004 \pm$ & $0.003 \pm$ & $0.013 \pm$ \\
\hline & & 1.00 & 0.03 & 0.08 & 0.00 & 0.05 & 0.09 \\
\hline & \multirow[t]{3}{*}{ Infection } & $16.655 \pm$ & $0.016 \pm$ & $0.078 \pm$ & $0.008 \pm$ & $0.006 \pm$ & $0.026 \pm$ \\
\hline & & 3.00 & 0.05 & 0.05 & 0.04 & 0.00 & 0.04 \\
\hline & & $10.951 \pm$ & $0.011 \pm$ & $0.051 \pm$ & $0.005 \pm$ & $0.004 \pm$ & $0.017 \pm$ \\
\hline \multirow{9}{*}{$\begin{array}{l}\text { Pessu } \\
\text { Market }\end{array}$} & Erectile dysfunction & 1.06 & 0.08 & 0.06 & 0.01 & 0.01 & 0.02 \\
\hline & \multirow[t]{2}{*}{ Rheumatism } & $19.365 \pm$ & $0.019 \pm$ & $0.090 \pm$ & $0.009 \pm$ & $0.007 \pm$ & $0.030 \pm$ \\
\hline & & 1.07 & 0.07 & 0.01 & 0.03 & 0.05 & 0.01 \\
\hline & \multirow[t]{2}{*}{ Malaria } & $16.296 \pm$ & $0.016 \pm$ & $0.076 \pm$ & $0.007 \pm$ & $0.006 \pm$ & $0.026 \pm$ \\
\hline & & 1.01 & 0.09 & 0.08 & 0.01 & 0.07 & 0.03 \\
\hline & \multirow[t]{2}{*}{ Typhoid } & $21.057 \pm$ & $0.021 \pm$ & $0.098 \pm$ & $0.010 \pm$ & $0.007 \pm$ & $0.033 \pm$ \\
\hline & & 3.02 & 0.00 & 0.05 & 0.08 & 0.09 & 0.10 \\
\hline & \multirow[t]{2}{*}{ Infection } & $9.005 \pm$ & $0.009 \pm$ & $0.042 \pm$ & $0.004 \pm$ & $0.003 \pm$ & $0.014 \pm$ \\
\hline & & 1.04 & 0.00 & 0.03 & 0.00 & 0.00 & 0.09 \\
\hline \multirow{10}{*}{$\begin{array}{l}\text { Effurun } \\
\text { Market }\end{array}$} & \multirow[t]{2}{*}{ Erectile dysfunction } & $9.419 \pm$ & $0.009 \pm$ & $0.044 \pm$ & $0.004 \pm$ & $0.003 \pm$ & $0.015 \pm$ \\
\hline & & 1.08 & 0.10 & 0.10 & 0.03 & 0.05 & 0.10 \\
\hline & \multirow[t]{2}{*}{ Rheumatism } & $11.745 \pm$ & $0.011 \pm$ & $0.055 \pm$ & $0.005 \pm$ & $0.004 \pm$ & $0.018 \pm$ \\
\hline & & 1.10 & 0.11 & 0.07 & 0.05 & 0.05 & 0.09 \\
\hline & \multirow[t]{2}{*}{ Malaria } & $21.904 \pm$ & $0.021 \pm$ & $0.102 \pm$ & $0.010 \pm$ & $0.007 \pm$ & $0.034 \pm$ \\
\hline & & 3.04 & 0.02 & 0.11 & 0.01 & 0.03 & 0.00 \\
\hline & \multirow[t]{2}{*}{ Typhoid } & $7.407 \pm$ & $0.007 \pm$ & $0.035 \pm$ & $0.003 \pm$ & $0.003 \pm$ & $0.012 \pm$ \\
\hline & & 2.06 & 0.08 & 0.11 & 0.02 & 0.08 & 0.03 \\
\hline & \multirow[t]{2}{*}{ Infection } & $8.507 \pm$ & $0.008 \pm$ & $0.040 \pm$ & $0.004 \pm$ & $0.003 \pm$ & $0.013 \pm$ \\
\hline & & 1.09 & 0.04 & 0.04 & 0.03 & 0.01 & 0.04 \\
\hline \multirow{10}{*}{$\begin{array}{l}\text { Okere } \\
\text { Market }\end{array}$} & \multirow[t]{2}{*}{ Erectile dysfunction } & $6.085 \pm$ & $0.006 \pm$ & $0.028 \pm$ & $0.003 \pm$ & $0.002 \pm$ & $0.010 \pm$ \\
\hline & & 1.00 & 0.00 & 0.10 & 0.00 & 0.00 & 0.03 \\
\hline & \multirow[t]{2}{*}{ Rheumatism } & $7.725 \pm$ & $0.008 \pm$ & $0.036 \pm$ & $0.003 \pm$ & $0.003 \pm$ & $0.012 \pm$ \\
\hline & & 1.07 & 0.01 & 0.01 & 0.00 & 0.00 & 0.05 \\
\hline & Malaria & $9.906 \pm$ & $0.010 \pm$ & $0.046 \pm$ & $0.004 \pm$ & $0.003 \pm$ & $0.016 \pm$ \\
\hline & & 1.07 & 0.07 & 0.02 & 0.01 & 0.01 & 0.02 \\
\hline & Typhoid & $19.576 \pm$ & $0.019 \pm$ & $0.091 \pm$ & $0.009 \pm$ & $0.007 \pm$ & $0.031 \pm$ \\
\hline & & 2.05 & 0.09 & 0.04 & 0.03 & 0.06 & 0.03 \\
\hline & Infection & $11.419 \pm$ & $0.011 \pm$ & $0.053 \pm$ & $0.005 \pm$ & $0.004 \pm$ & $0.018 \pm$ \\
\hline & & 2.10 & 0.06 & 0.04 & 0.06 & 0.03 & 0.01 \\
\hline & Erectile dysfunction & $9.062 \pm$ & $0.009 \pm$ & $0.042 \pm$ & $0.004 \pm$ & $0.003 \pm$ & $0.014 \pm$ \\
\hline Igbudu & & 1.05 & 0.05 & 0.03 & 0.00 & 0.08 & 0.04 \\
\hline
\end{tabular}


Physicochemical and Microbiological Evaluation of Agbo (herbal decoction) Sold in Some Parts of Warri Metropolis, Delta-State, Nigeria

\begin{tabular}{crrrrrrr} 
Market & Rheumatism & $8.519 \pm$ & $0.008 \pm$ & $0.040 \pm$ & $0.004 \pm$ & $0.003 \pm$ & $0.013 \pm$ \\
& & 1.02 & 0.00 & 0.01 & 0.07 & 0.07 & 0.09 \\
& Malaria & $15.926 \pm$ & $0.016 \pm$ & $0.074 \pm$ & $0.007 \pm$ & $0.005 \pm$ & $0.025 \pm$ \\
& 2.03 & 0.02 & 0.06 & 0.01 & 0.05 & 0.03 \\
& & $14.603 \pm$ & $0.014 \pm$ & $0.068 \pm$ & $0.007 \pm$ & $0.005 \pm$ & $0.023 \pm$ \\
& Typhoid & 3.10 & 0.01 & 0.07 & 0.02 & 0.06 & 0.10 \\
& & $10.370 \pm$ & $0.010 \pm$ & $0.048 \pm$ & $0.005 \pm$ & $0.004 \pm$ & $0.016 \pm$ \\
& 1.10 & 0.03 & 0.02 & 0.05 & 0.02 & 0.01 \\
\hline
\end{tabular}

Key: Fe - Iron, Pb - Lead, Cd - Cadmium, Hg - Mercury, As - Arsenic $\mathrm{Cr}$ - Chromium

Table 3: Total viable bacterial count, coliform count and fungi count (mean $\left.\pm S . D \times 10^{4} \mathrm{cfu} / \mathrm{ml}\right)$

\begin{tabular}{|c|c|c|c|c|}
\hline Sample source & Sample type & $\begin{array}{l}\text { Total bacterial } \\
\text { count }\end{array}$ & Coliform count & Fungi count \\
\hline \multirow[t]{5}{*}{ Main Market } & Erectile dysfunction & $25 \pm 2.05^{\mathrm{b}}$ & $0.17 \pm 0.09^{a}$ & $35 \pm 0.15^{\mathrm{c}}$ \\
\hline & Rheumatism & $32 \pm 1.08^{c}$ & $0.19 \pm 2.03^{a}$ & $20 \pm 1.00^{\mathrm{b}}$ \\
\hline & Malaria fever & $34 \pm 0.06^{c}$ & $0.18 \pm 1.03^{\mathrm{a}}$ & $37 \pm 1.50^{c}$ \\
\hline & Typhoid fever & $38 \pm 3.03^{c}$ & $0.23 \pm 1.08^{\mathrm{a}}$ & $40 \pm 1.00^{\mathrm{c}}$ \\
\hline & General Infection & $28 \pm 2.02^{b}$ & $0.20 \pm 1.00^{\mathrm{a}}$ & $31 \pm 2.01^{\mathrm{c}}$ \\
\hline \multirow[t]{5}{*}{ Pessu market } & Erectile dysfunction & $35 \pm 1.05^{\mathrm{c}}$ & $0.26 \pm 1.04^{\mathrm{a}}$ & $29 \pm 1.09^{b}$ \\
\hline & Rheumatism & $29 \pm 2.03^{\mathrm{b}}$ & $0.15 \pm 0.05^{\mathrm{a}}$ & $32 \pm 0.51^{\mathrm{c}}$ \\
\hline & Malaria fever & $30 \pm 1.01^{c}$ & $0.16 \pm 2.04^{\mathrm{a}}$ & $38 \pm 1.07^{\mathrm{c}}$ \\
\hline & Typhoid fever & $28 \pm 1.03^{b}$ & $0.29 \pm 1.01^{\mathrm{a}}$ & $31 \pm 1.00^{c}$ \\
\hline & General Infection & $31 \pm 0.09^{c}$ & $0.30 \pm 0.11^{\mathrm{a}}$ & $35 \pm 0.51^{\mathrm{c}}$ \\
\hline \multirow[t]{5}{*}{ Effurun market } & Erectile dysfunction & $32 \pm 1.03^{c}$ & $0.18 \pm 0.07^{\mathrm{a}}$ & $29 \pm 2.06^{b}$ \\
\hline & Rheumatism & $25 \pm 2.05^{b}$ & $0.19 \pm 2.09^{\mathrm{a}}$ & $30 \pm 1.03^{b}$ \\
\hline & Malaria fever & $44 \pm 0.06^{\mathrm{c}}$ & $0.17 \pm 2.00^{\mathrm{a}}$ & $38 \pm 1.51^{\mathrm{c}}$ \\
\hline & Typhoid fever & $40 \pm 1.00^{\mathrm{c}}$ & $0.21 \pm 1.03^{\mathrm{a}}$ & $49 \pm 1.05^{c}$ \\
\hline & General Infection & $42 \pm 2.01^{\mathrm{c}}$ & $0.22 \pm 1.00^{\mathrm{a}}$ & $35 \pm 0.09^{c}$ \\
\hline \multirow[t]{5}{*}{ Okere Market } & Erectile dysfunction & $31 \pm 2.03^{c}$ & $0.18 \pm 2.08^{\mathrm{a}}$ & $28 \pm 0.50^{b}$ \\
\hline & Rheumatism & $38 \pm 1.02^{c}$ & $0.27 \pm 1.06^{\mathrm{a}}$ & $37 \pm 1.04^{c}$ \\
\hline & Malaria fever & $35 \pm 1.06^{\mathrm{c}}$ & $0.15 \pm 3.05^{\mathrm{a}}$ & $31 \pm 0.51^{c}$ \\
\hline & Typhoid fever & $30 \pm 3.00^{\mathrm{c}}$ & $0.16 \pm 2.09^{a}$ & $31 \pm 0.50^{c}$ \\
\hline & General Infection & $33 \pm 0.09^{c}$ & $0.21 \pm 1.10^{\mathrm{a}}$ & $40 \pm 2.00^{c}$ \\
\hline \multirow[t]{5}{*}{ Igbudu market } & Erectile dysfunction & $36 \pm 1.00^{\mathrm{c}}$ & $0.16 \pm 1.08^{\mathrm{a}}$ & $25 \pm 1.06^{\mathrm{b}}$ \\
\hline & Rheumatism & $33 \pm 2.07^{c}$ & $0.19 \pm 0.10^{\mathrm{a}}$ & $35 \pm 0.11^{\mathrm{c}}$ \\
\hline & Malaria fever & $38 \pm 1.03^{c}$ & $0.15 \pm 0.51^{\mathrm{a}}$ & $48 \pm 3.01^{\mathrm{c}}$ \\
\hline & Typhoid fever & $37 \pm 0.08^{c}$ & $0.18 \pm 0.00^{\mathrm{a}}$ & $46 \pm 2.50^{c}$ \\
\hline & General Infection & $34 \pm 1.03^{\mathrm{c}}$ & $0.17 \pm 2.01^{\mathrm{a}}$ & $36 \pm 1.04^{\mathrm{c}}$ \\
\hline
\end{tabular}

The superscripts $\mathrm{b}=\mathrm{c}$ (the difference between $\mathrm{b}$ and $\mathrm{c}$ is not statistically significant)

But $b \neq a$ and $c \neq a$ (the difference between $b \& a$ and also $c \& a$ are statistically significant $P<0.05$ )

$\mathrm{b}=\mathrm{c} \neq \mathrm{a} *$ ( $\mathrm{a}$ is significantly different from $\mathrm{b}$ and $\mathrm{c}$ at $P<0.05$ )

Table 4: bacterial and fungal isolates

\begin{tabular}{lll}
\hline Sample source & Identified bacterial isolates & Identified fungal isolates \\
\hline Main Market & Salmonella species & Aspergillus species \\
& Klebsiella species & yeast species \\
& Eschericha coli & Penicillium \\
Staphylococcus aureus & Botrytis species \\
& & \\
Pessu market & Klebsiella species & $\begin{array}{l}\text { Aspergillus species } \\
\text { yeast species } \\
\end{array}$ \\
& Staphylococcus aureus & Mucor
\end{tabular}




\begin{tabular}{|c|c|c|}
\hline Effurun market & $\begin{array}{l}\text { Klebsiella species } \\
\text { Eschericha coli } \\
\text { Salmonella species } \\
\text { Staphylococcus aureus }\end{array}$ & $\begin{array}{l}\text { Aspergillus species } \\
\text { Penicillium } \\
\text { Fusarium spp. }\end{array}$ \\
\hline Okere Market & $\begin{array}{l}\text { Klebsiella species } \\
\text { Proteus mirabilis } \\
\text { Eschericha coli } \\
\text { Staphylococcus aureus }\end{array}$ & $\begin{array}{l}\text { Aspergillus species } \\
\text { yeast species } \\
\text { Mucor } \\
\text { Fusarium spp. }\end{array}$ \\
\hline Igbudu market & $\begin{array}{l}\text { Enterobacter species } \\
\text { Staphylococcus aureus } \\
\text { Proteus mirabilis }\end{array}$ & $\begin{array}{l}\text { Aspergillus species } \\
\text { yeast species } \\
\text { Penicillium } \\
\text { Mucor } \\
\text { Botrytis species } \\
\text { Geotrichum spp } \\
\text { Phoma spp. }\end{array}$ \\
\hline
\end{tabular}

Table 5: Antibiotics susceptibility of bacterial isolates

\begin{tabular}{|c|c|c|c|c|c|c|c|c|c|c|}
\hline \multicolumn{11}{|c|}{ Inhibition zone diameter (mean \pm S.D mm) } \\
\hline $\begin{array}{l}\text { Bacterial } \\
\text { Isolates }\end{array}$ & $\begin{array}{l}\text { OFX } \\
(10 \mu g)\end{array}$ & $\begin{array}{l}\text { PEF } \\
(10 \mu g)\end{array}$ & $\begin{array}{l}\text { CPF } \\
(10 \mu \mathrm{g})\end{array}$ & $\begin{array}{l}\mathrm{AU} \\
(30 \mu \mathrm{g})\end{array}$ & $\begin{array}{l}\mathrm{CN} \\
(10 \mu \mathrm{g})\end{array}$ & $\begin{array}{l}\text { S } \\
(30 \mu \mathrm{g})\end{array}$ & $\begin{array}{l}\text { CEP } \\
(10 \mu \mathrm{g})\end{array}$ & $\begin{array}{l}\text { NA } \\
(30 \mu g)\end{array}$ & $\begin{array}{l}\text { SXT } \\
(30 \mu \mathrm{g})\end{array}$ & $\begin{array}{l}\text { PN } \\
(30 \mu \mathrm{g})\end{array}$ \\
\hline S. aureus & $\begin{array}{l}18 \pm 1.0 \\
(\mathrm{R})\end{array}$ & $\begin{array}{l}20 \pm 3.1 \\
\text { (I) }\end{array}$ & $\begin{array}{l}18 \pm 3.0 \\
\text { (I) }\end{array}$ & $\begin{array}{l}32 \pm 3.1 \\
(\mathrm{~S})\end{array}$ & $\begin{array}{l}20 \pm 1.1 \\
(\mathrm{~S})\end{array}$ & $\begin{array}{l}14 \pm 0.0 \\
(\mathrm{R})\end{array}$ & $\begin{array}{l}23 \pm 3.1 \\
(\mathrm{~S})\end{array}$ & - & $\begin{array}{l}18 \pm 1.1 \\
(\mathrm{R})\end{array}$ & $\begin{array}{l}28 \pm 1.3 \\
(\mathrm{R})\end{array}$ \\
\hline E. coli & $\begin{array}{l}20 \pm 3.3 \\
\text { (I) }\end{array}$ & $\begin{array}{l}16 \pm 1.0 \\
(\mathrm{R})\end{array}$ & $\begin{array}{l}24 \pm 0.0 \\
(\mathrm{~S})\end{array}$ & $\begin{array}{l}21 \pm 1.4 \\
\text { (I) }\end{array}$ & $\begin{array}{l}21 \pm 2.3 \\
(\mathrm{~S})\end{array}$ & $\begin{array}{l}12 \pm 1.1 \\
(\mathrm{R})\end{array}$ & $\begin{array}{l}14 \pm 0.0 \\
(\mathrm{R})\end{array}$ & $\begin{array}{l}24 \pm 3.0 \\
(S)\end{array}$ & - & $\begin{array}{l}18 \pm 0.0 \\
(\mathrm{R})\end{array}$ \\
\hline $\begin{array}{l}\text { Klebsiella } \\
\text { spp. }\end{array}$ & $\begin{array}{l}19 \pm 1.1 \\
\text { (I) }\end{array}$ & $\begin{array}{l}18 \pm 3.4 \\
\text { (I) }\end{array}$ & $\begin{array}{l}25 \pm 3.1 \\
(\mathrm{~S})\end{array}$ & $\begin{array}{l}16 \pm 2.3 \\
(\mathrm{R})\end{array}$ & $\begin{array}{l}28 \pm 1.6 \\
(\mathrm{~S})\end{array}$ & $\begin{array}{l}16 \pm 3.1 \\
\text { (I) }\end{array}$ & $\begin{array}{l}14 \pm 1.0 \\
(\mathrm{R})\end{array}$ & $\begin{array}{l}16 \pm 2.4 \\
(\mathrm{R})\end{array}$ & $\begin{array}{l}18 \pm 1.3 \\
(\mathrm{R})\end{array}$ & $\begin{array}{l}12 \pm 2.6 \\
(\mathrm{R})\end{array}$ \\
\hline $\begin{array}{l}\text { Salmonella } \\
\text { spp. }\end{array}$ & $\begin{array}{l}18 \pm 3.0 \\
\text { (I) }\end{array}$ & $\begin{array}{l}18 \pm 2.2 \\
\text { (I) }\end{array}$ & $\begin{array}{l}27 \pm 0.8 \\
(S)\end{array}$ & $\begin{array}{l}16 \pm 0.1 \\
(\mathrm{R})\end{array}$ & $\begin{array}{l}18 \pm 1.1 \\
\text { (I) }\end{array}$ & $\begin{array}{l}14 \pm 2.0 \\
(\mathrm{R})\end{array}$ & $\begin{array}{l}16 \pm 3.4 \\
(\mathrm{R})\end{array}$ & - & - & $\begin{array}{l}14 \pm 0.5 \\
(\mathrm{R})\end{array}$ \\
\hline P. mirabilis & $\begin{array}{l}20 \pm 1.5 \\
\text { (I) }\end{array}$ & $\begin{array}{l}20 \pm 0.8 \\
\text { (I) }\end{array}$ & $\begin{array}{l}26 \pm 1.8 \\
(\mathrm{~S})\end{array}$ & - & $\begin{array}{l}25 \pm 3.3 \\
(\mathrm{~S})\end{array}$ & $\begin{array}{l}16 \pm 2.4 \\
(\mathrm{R})\end{array}$ & $\begin{array}{l}18 \pm 3.0 \\
(\mathrm{R})\end{array}$ & $\begin{array}{l}16 \pm 0.0 \\
(\mathrm{R})\end{array}$ & - & $\begin{array}{l}16 \pm 3.3 \\
(\mathrm{R})\end{array}$ \\
\hline $\begin{array}{l}\text { Enteobacter } \\
\text { spp. }\end{array}$ & $\begin{array}{l}16 \pm 0.0 \\
(\mathrm{R})\end{array}$ & $\begin{array}{l}23 \pm 0.8 \\
(\mathrm{~S})\end{array}$ & $\begin{array}{l}19 \pm 3.3 \\
\text { (I) }\end{array}$ & $\begin{array}{l}24 \pm 0.0 \\
(\mathrm{~S})\end{array}$ & $\begin{array}{l}22 \pm 1.3 \\
(\mathrm{~S})\end{array}$ & - & $\begin{array}{l}18 \pm 1.5 \\
\text { (I) }\end{array}$ & $\begin{array}{l}20 \pm 3.5 \\
(\mathrm{R})\end{array}$ & - & $\begin{array}{l}14 \pm 1.4 \\
(\mathrm{R})\end{array}$ \\
\hline
\end{tabular}

Key:

OFX=Ofloxacin, $\quad \mathrm{PEF}=$ Pefloxacine, $\quad \mathrm{CPX}=$ Ciprofloxacin, $\mathrm{AU}=$ Augmentin, $\quad \mathrm{CN}=\mathrm{Gentamycin}, \quad \mathrm{S}=$ Streptomycin, $\mathrm{CEP}=$ Ceporex, $\quad \mathrm{NA}=$ Nalidixic Acid, SXT=Septrin, $\mathrm{PN}=$ Ampicillin,

No inhibition, $\mathrm{S}=$ Susceptible, $\mathrm{I}=$ Intermediate, $\mathrm{R}=$ Resistant

Note that IZD's were interpreted using Kirby-Bauer antibiogram table. 


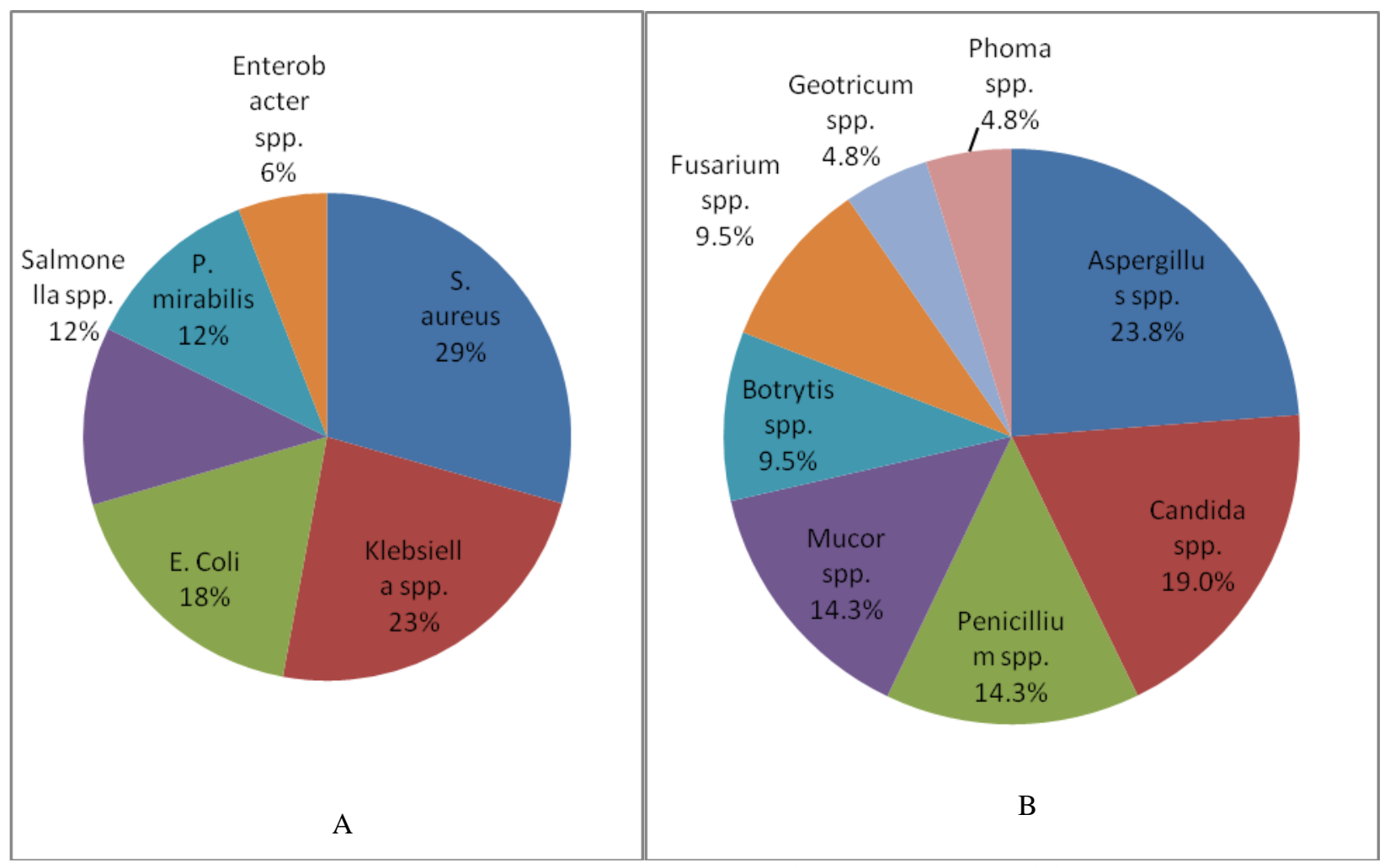

Figure 1: Percentage occurrence of bacterial isolates (A) Fungal isolates (B)

The $\mathrm{pH}$ values of the various agbo samples ranges from 3.38 \pm 1.05 to $7.23 \pm 1.02$ as shown in table 1 . These $\mathrm{pH}$ values are in the acidic region except for one having $\mathrm{pH}$ of $7.23 \pm 1.02$ which is in basic region of the $\mathrm{pH}$ scale. Richard (2017) reported that the human body needs to take in the right amount of acidic and alkalizing nutrients to maintain a healthy $\mathrm{pH}$ balance. The recommended daily intake should be $20 \%$ of acidic and $80 \%$ alkalizing. He stated further that if the body acid/alkaline balance is not maintained, the body has to compensate by robbing minerals from other parts of the body such as the bones, joints, muscles, gall bladder and mucosal lining of the digestive tract. Thus, when the body robs calcium from the bones, one can develop a weak back and if left untreated, this can develop into osteoporosis (low bone density). Therefore, people who regularly consume those agbo samples are at risk of developing acidic/alkalinic imbalance which can result to various diseases

Table 2. Shows concentration of heavy metals in the various agbo samples sold in Warri. Concentration of Iron ranges from $6.085 \pm 1.00-21.904 \pm 3.04 \mathrm{mg} / \mathrm{l}$, Lead ranges from $0.006 \pm 0.00-0.021 \pm 0.08 \mathrm{mg} / \mathrm{l}, \mathrm{Cadmium}$ ranges from $0.028 \pm 0.10-0.102 \pm 0.11 \mathrm{mg} / \mathrm{l}$, Mercury ranges from 0.003 $\pm 0.00-0.010 \pm 0.08 \mathrm{mg} / \mathrm{l}$, Arsenic ranges from $0.002 \pm 0.00$ $-0.007 \pm 0.05 \mathrm{mg} / \mathrm{l}$ and Chromium ranges from $0.012 \pm 0.03$ $0.034 \pm 0.01 \mathrm{mg} / \mathrm{l}$ respectively. The World Health Organization (WHO) limits for Arsenic, Cadmium, Lead and Chromium in herbal preparation are $0.01 \mathrm{mg} / 1,0.003 \mathrm{mg} / \mathrm{l}$, $0.001 \mathrm{mg} / \mathrm{l}$ and $0.02 \mathrm{mg} / \mathrm{l}$ respectively, while $0.002 \mathrm{mg} / \mathrm{l}$ is the limit for Mercury set by the Food and Drug Administration (FDA) and Environmental Protection Agency (EPA) (Osadolor et al, 2015). The concentration of heavy metals in all the agbo samples are above the limit. Persistent users may accumulate these heavy metals to levels that are toxic to organs within the body with time. According to Osadolor et al., (2015), adult with high blood lead levels $(0.04 \mathrm{mg} / \mathrm{l})$ may have impaired haemsynthesis, chronic kidney disease and impairment of cognitive function. Increased exposure to mercury can alter brain functions and lead to shyness, tremors, memory problems, irritability, and changes in vision or hearing (Monisha et al., 2014). Ingestion of large amounts of arsenic can lead to gastrointestinal symptoms such as severe vomiting, disturbances of the blood and circulation, damage to the nervous system and eventually death (Manju, 2015). Iron produces free radicals that results in cellular damage, mutation and malignant transformations which in turn can cause an array of diseases (Monisha et al, 2014). Cadmium damages a specific structure of the functional unit of the kidney.

Table 3 shows the estimation of the total viable bacterial count, total coliform counts and total fungi count of the various agbo samples on nutrient agar, MacConkey agar and potato Dextrose agar. The mean total viable count ranges between $25 \pm 1.03 \times 10^{4}-44 \pm 0.06 \times 10^{4} \mathrm{cfu} / \mathrm{ml}$, total coliforn count ranges between $0.15 \pm 0.51 \times 10^{4}-0.30 \pm 0.11$ $\mathrm{X} 10^{4} \mathrm{cfu} / \mathrm{ml}$ and total fungal count ranges between $25 \pm 1.06$ - $49 \pm 1.05 \times 10^{4} \mathrm{cfu} / \mathrm{ml}$ respectively. The microbial counts detected were in the order of $10^{4} \mathrm{cfu} / \mathrm{ml}$. The World Health Organization (WHO), British Pharmacopoeia and the United State Pharmacopoeia have recommended tolerable microbial limits in non - sterile pharmaceutical products which include $10^{7} \mathrm{cfu} / \mathrm{ml}$ bacteria and $10^{5} \mathrm{cfu} / \mathrm{ml}$ fungi. All the agbo samples were able to meet this limit. However, the detection of bacteria such as $S$. aureus andcoliforms which are food borne pathogens in the samples shows high level of contamination from different sources which makes the quality of the samples unacceptable. The occurrence of $S$. aureus in majority of the samples could be from the nose where it is commonly found, hands, skin and clothing of 
handlers. Coliform contamination could result from the water used in preparing or diluting the agbo and poor personal hygiene during preparation. Some of the fungi species of Aspergillus are known to produce mycotoxins that are harmful to man and as such, their incidence in agbo is undesirable. The presence of these fungi isolated may be due to poor harvesting conditions and post harvestingpreservation techniques used for the herbs. These observations are in agreement with the work of Odedara and Memuletiwon, (2014) who recorded total bacterial count of $8.5 \times 10^{5} \mathrm{cfu} / \mathrm{ml}$

Table 4 shows the distribution of bacterial and fungal pathogens isolated from agbo samples sold in Warri metropolis. Six bacteria and eight fungi were isolated.

Figure 1 showsthepercentage of occurrence of the various bacteria and fungi isolates. S.auerus (29.4\%) was the predominant bacteria. This was followed by Klebsiella species $(23.5 \%)$, E.coli (17.6\%), Salmonella species (11.8\%), Proteus mirabilis (11.8\%) and Enterobacter species $(5.9 \%)$. Aspergillus species (23.8) was the predominant fungi. This was followed by Candida species (19.0\%), penicillium spp (14.3\%), Mucor (14.3\%), Botrytis (9.5\%), Fusarium spp (9.5\%), geotrichum spp (4.8\%) and Phoma spp (4.8\%). This observation is in agreement with the work of MacDonald et al, (2015) who reported isolating fungal species such as Fusarium spp, Aspergillus spp, Penicillium spp, Geotrichum spp, and Mucor from poly - herbal products in Lagos. Esimone et al (2001) also reported the presence of bacteria such as E. coli, Klebsiella spp, Proteus spp, Streptococus spp and Staphylococcus spp from herbal preparations marketed in South East Nigeria

Table 5 shows the antibiotics sensitivity of the various bacteria isolated from the agbo samples. All the isolates were resistant to ampicillin. S.areus was susceptible to augmentin, gentamycin and ceporex, E. coli was susceptible to ciprofloxacin, gentamycin and nalidixic acid. Klebsiella spp was susceptible to ciprofloxacin and getamycin, Salmonella spp was susceptible to ciprofloxacin, P.mirabilis was susceptible to ciprofloxacin and gentamycin, and Enterobacter spp was susceptible to pefloxacine, augmentin and gentamycin. These antibiotics have been in the Nigerian market for a long time and might have been exposed to use and abuse which might account for the levels of resistance observed in the study. Therefore, gentamycin and ciprofloxacin are antibiotics options that can be used to treat bacterial infections acquired from the consumption of agbo sold in Warri, Delta state.

\section{CONCLUSION}

This study has revealed that agbo sold in Warri metropolis contain relatively high amounts of heavy metals above the limit and by implication, frequent consumption may increase the body burden of these metals, which have been reported to be toxic to the body. The study also reveal the presence of pathogenic bacteria and fungi in the agbo samples which could have resulted from contaminated soils, plants and its products, preparation processes, quality of water, storage containers and processing equipment. This stands as a great threat to human health as these organisms can cause diseases. The study also revealed the antibiotic sensitivity of the bacterial isolates. The presence of antibiotic resistant organisms raises concerns to health as it may implicate the clinical management of diseases caused by the resistant organism in human. The use of agbo as an alternative drug could be a source of multiple antibiotics resistance microorganisms in consumers. Efforts on the part of producers should be made to enhance proper hygienic conditions in all the preparation processes starting from plant collection, processing, packaging and storage in order to reduce incidence of pathogenic microorganisms and spread of resistance strains

\section{REFERENCES}

[1]Barnett H.L. and Hunter, B.B. (1998). Illustrated Genera of Imperfect Fungi ( $4^{\text {th }}$ Edition). Published by Amer Phytopathological Society. Pp 106,130

[2]Bauer, A.M., Kirby, W.M.M., Shermis, T.C. and Truck, M. (1966). Antibiotics Susceptibility Testing by A Standard Single Disk Method. Am. J. Clin. Pathol. 45:493 - 496

[3]Buchanna, R.E. and Gibbon, N.E.(1974). Bergy's Manual of Determination Bacteriology, Williams and Wilkin Baltimore, USA.

[4]Caldas L.L. Machado (2004). Cadmium, Mercury and Lead in medicinal herbs in Brazil. Food and Chemical Toxicology. Vol 42, $599-603 p p$

[5]Christen, G.L., Davidson, P.M., McAllister, J.S. and Roth, L.A. (1992). Coliform and other indicator bacteria in: Standard Method for the Examination of Dairy product, Marshall, T.R. (Ed.) American Public Health Association, Washington, DC. Pp: $247-267$

[6]Esimone C.O; Chach K.F, and Ikejide S.C. (2001). Microbiological quality of herbal preparations marked in South East Nigeria. Journal of natural remedies. 2: 42-48.

[7]Falodun A. and Imieje V. (2013). Herbal Medicine in Nigeria; holistic overview. Nigeria journal of science and environment. 12(1)

[8] Macdonald Idu, Ouvakporie Uvo Oghale, Adeola Jimoh (2015). Heavy metals contamination of some poly herbal products from Lagos State, Nigeria. Journal of Ayurvedic and Herbal medicine. 1(2): $45-50 \mathrm{pp}$

[9] Macdonald I., Ouvakporie U. O., Adeola J. (2015). Microbial load of some poly- herbal products from Lagos State, Nigeria. International Journal of Ethnobiology and Ethnomedicine. 1: issue 1

[10] Manju M. (2015). Effects of heavy metals on human health. International Journal of research Granthalayah. 1 - 7pp

[11] Monisha J. T.T., Naresh A. Blessing B., Mathew K. and Beeregowda N. (2014). Toxicity, mechanism and health effects of some heavy metals. Journal of interdisciplinary toxicology. Vol 7(2): $60-72 p p$

[12] Odedera, O.O. and Memuletiwon, E.J. (2014). Microbial quality of some locally consumed herbal concoctions in Abeokuta metropolis, Nigeria. Journal of National Science, Engineering and Technology. $13: 58-66$

[13] Ola I.O.. Omomowo I.O., Anina D.A., Majolagbe N.O. and Oladipo E.K. (2013). Bacteriological evaluation and antimicrobial sensitivity test of common herbal concoction in ogbomoso Metropolis. African journal of microbiology research 7(48):5479-5483.

[14] Olmedo, P., Pedro F., Fernado, G., Antonio, H. and Antonio, P. (2010). Biomonitorization of cadmium, chromium, manganese, nickel and lead in whole blood, urine, axillary hair and saliva in an occupational exposed population.

[15] Oluyege, J.O. and Oluyege, J.O. (2010). Microbial contamination of some hawked herbal products in Ado - Ekiti, Nigeria. Continental Journal Microbiology 4:8-14

[16] Oluyemi J., Yinusa M.,, Abdullateef R. and Adejoke J. (2016) Utilization of herbal medicine among inhanitants of an urban centre in North - central Nieria. Algerian journal of natural products, 4(3): 367 $-378$

[17] Osadolor, H.B., Igharo, O.G., Onuyon-Adatire, A.E., David, O.A. and Akpomiemie, V.O. (2015). Evaluation of Levels of Toxic Metals in commonly used Herbal Medicines in Bennin City, South- South, Nigeria. Annala of Biomedical Sciences 14:82-89

[18] Richard K.C. (2017). Microbial and Heavy Contaminations in selected Herbal Medicinal Products sold in Nairobi, Kenya. International Journal of Pharmaceutical Research. 8(1): 135- 138

[19] Stafford G.I, Redersen M.E., Van Stadew and Jager K. (2008). Review on plants with CNS - effects used in traditional South African medicine against mental diseases. Journal of Ethropharmacology. $119(3) .513-57 \mathrm{pp}$ 